\title{
Image Dominant Colors Estimation and Color Reduction Via a New Self-growing and Self-organized Neural Gas*
}

\author{
A. Atsalakis, N. Papamarkos ${ }^{1}$, and I. Andreadis \\ ${ }^{1}$ Image Processing and Multimedia Laboratory, \\ Department of Electrical \& Computer Engineering, \\ Democritus University of Thrace, \\ 67100 Xanthi, Greece \\ papamark@ee.duth.gr
}

\begin{abstract}
A new method for the reduction of the number of colors in a digital image is proposed. The new method is based on the development of a new neural network classifier that combines the advantages of the Growing Neural Gas (GNG) and the Kohonen Self-Organized Feature Map (SOFM) neural networks. We call the new neural network: Self-Growing and Self-Organized Neural Gas (SGONG). Its main advantage is that it defines the number of the created neurons and their topology in an automatic way. Besides, a new method is proposed for the Estimation of the Most Important of already created Classes (EMIC). The combination of SGONG and EMIC in color images results in retaining the isolated and significant colors with the minimum number of color classes. The above techniques are able to be fed by both color and spatial features. For this reason a similarity function is used for vector comparison. To speed up the entire algorithm and to reduce memory requirements, a fractal scanning sub-sampling technique is used. The method is applicable to any type of color images and it can accommodate any type of color space.
\end{abstract}

\section{Introduction}

The reduction of the number of colors in digital images is an active research area. True type color images consist of more than 16 million of different colors. The image color reduction is an important task for presentation, transmission, segmentation and compression of color images. The proposed method can be considered as a Color Quantization (CQ) technique. The goal of the CQ techniques is to reduce the number of colors in an image in a way that minimizes the perceived difference between the original and the quantized image. Several techniques have been proposed for CQ which can be classified in the following three major categories. Firstly, there is a class of techniques that are based on splitting algorithms. According to those approaches, the color space is divided into disjointed regions by consecutive splitting up the color space. The methods of median-cut [1] and variance-based algorithm [2] belong to this category. The method of Octree [3] is based on splitting the color space to smaller

* This work is supported by the project PYTHAGORAS 1249-6. 
cubes. An optimized splitting technique is proposed by $\mathrm{Wu}$ [4] who utilizes the principal component analysis to split optimally the original color space. The second major class of CQ techniques is based on cluster analysis. Techniques in this category attempt to find the optimal palette by using vector classifiers. In this category belong methods like ACR [5], FOSART [6], Fuzzy ART [7] and FCM [8]. As it is noticed by Buhmann et al. [9], one of the basic disadvantages of the most classic CQ approaches is the fact that they neglect spatial, i.e. contextual, information. In order to overcome this disadvantage, the color reduction problem must be considered as a clustering problem with the input vectors describing not only the color information but also extra spatial features derived from the neighboring area of each pixel [10-11]. Artificial neural networks are very efficient approaches to create powerful vector classifiers and to solve clustering problems. A well-known unsupervised neural network classifier is the Kohonen SOFM [12]. This network consists of two separate layers of fully connected neurons, i.e. the input and the output layer. Although, the Kohonen SOFM performs topology preserving mapping, there is a major drawback: the dimensions of the input space and the output lattice of neurons are not always identical and, consequently, the structure of the input data is not always preserved in the output layer.

Several implementations of the Kohonen SOFM have been proposed for color reduction. Dekker [13] proposes a color reduction technique which is based on a Kohonen SOFM classifier. According to this approach, equal sized classes are produced. Papamarkos and Atsalakis propose a new technique according to which a Kohonen SOFM neural network is fed not only with the image gray-scale values but also with local spatial features extracted from the neighboring of the sampling pixels [10-11]. An extension to the methods mentioned above, is the Adaptive Color Reduction (ACR) technique [5]. This technique, by applying a tree-driven splitting and merging strategy, decomposes the initial image into a number of color planes. A two-stage color segmentation methodology based on a Kohonen SOFM network is also proposed by Huang et al. [14]. Ashikur et al. [15] propose a CQ technique by combining the SOFM with a supervised counter propagation network. With the exception of the ACR algorithm, all the techniques mentioned above have the same drawback: the final number of colors should be predefined.

The proposed color reduction technique uses a new Self-Growing and SelfOrganized Neural Gas (SGONG) network. We develop this neural classifier in order to combine the growing mechanism of the GNG algorithm [16] and the learning scheme of the Kohonen SOFM. Specifically, the learning rate and the influence of neighboring neurons are monotonically decreased with the time. Furthermore, at the end of each epoch, three criteria are applied that improve the mechanism of growing and the convergence efficiency of the network. These criteria define when a neuron must be removed or added to the output lattice. The proposed neural network is faster than the Kohonen SOFM. In contrast with the GNG classifier, a local counter is defined for each neuron that influences the learning rate of this neuron and the strength of its connections with the neighboring neurons. In addition, these local counters are also used to specify the convergence of the proposed neural network. The main advantage of the SGONG classifier is its ability to influence the final number of neurons by using three suitable criteria. Thus, in the color reduction problem, the proper number of the image's dominant colors can be adaptively determined. 
An extension to any vector quantization technique is proposed for Estimation of the Most Important of already created Classes (EMIC). In order to function the EMIC technique, a predefined number of classes and a set of vectors representative of data space is required. The proposed technique is based on Comparative Hebbian Rule CHR [17] and no heuristic parameters are adjusted in order to be applied.

Concluding, in this paper

- A new self-growing and self-organized neural network is introduced. This SGONG neural network has the following characteristics:

- faster than the Kohonen SOFM,

- the dimensions of the input space and the output lattice of neurons are always identical. Thus, the structure of neurons in the output layer approaches the structure of the input data,

- criteria are used to ensure fast converge of the neural network, detection is of isolated classes and automatically estimation the number of neurons in the output layer.

- The EMIC method is proposed for choosing efficiently very few numbers of classes in automatic way.

- Even though the quantized image is described with few colors, isolated and small color classes can be detected combining the SGONG and EMIC methods

- Except for color components, the above methods can also be fed by additional local spatial features.

- The color reduction results obtained are better than previous reported techniques.

The proposed color reduction technique was tested by using a variety of images and the results are compared with other similar techniques.

\section{Combination of Color and Spatial Features}

To simplify our approach, let us consider a digital image of $n$ pixels and the corresponding data set $X$, consisting of $n$ input vectors (feature vectors) $X_{k}$ :

$$
X_{k}=\left[f 1_{k}, f 2_{k}, f 3_{k}, g 1_{k}, g 2_{k}, g 3_{k}, z 1_{k}, z 2_{k}\right], k=1, \ldots, n
$$

where $X_{k} \in \mathfrak{R}^{D}$, with $D=8$ the dimensionality of the input space. Each input vector $X_{k}$ consists of the pixel's color components $f_{k}=\left[f 1_{k}, f 2_{k}, f 3_{k}\right]$ and additional spatial features $g_{k}=\left[g 1_{k}, g 2_{k}, g 3_{k}\right]$ and $z_{k}=\left[z 1_{k}, z 2_{k}\right]$ extracted from a neighborhood region of this pixel. In color images, using for example the RGB color space, the elements $f 1_{k}, f 2_{k}, f 3_{k} \in[0,255]$, express the intensity values of red, green and blue color components of the $k$ pixel. Apart from the RGB color space, which is not perceptually uniform, more advantageous and perceptually uniform color spaces like CIE-L*a*b* or CIE-L*u* $\mathrm{v}^{*}$, can be used.

The neighborhood region of each pixel can be defined using a mask of $3 \times 3$ or $5 \times 5$ dimensions, where the central mask's element expresses the pixel's position. 
Depending on the spatial features used, the color of each pixel is associated with the spatial color characteristics of the neighboring pixels. The spatial feature vectors $g_{k}$ and $z_{k}$ can be derived from edge extraction, smoothing, noise reduction masks, min and max values, etc. Besides, the coordinates of each pixel can be used as additional features. According to the above analysis, color domain and spatial domain are concatenated in a joint domain of features. The vector $X_{k}$ combine vectors of different nature and thus their elements take values in different ranges. In order to compare efficiently two different vectors $X_{k}$ and $X_{m},(k, m=1, \ldots n)$ a similarity function $S\left(X_{k}, X_{m}\right)$ is defined which takes values in range [0,1] [18]. If $S\left(X_{k}, X_{m}\right)=1$ then the vectors $X_{k}$ and $X_{m}$ are considered equal.

$$
\begin{aligned}
& S\left(X_{k}, X_{m}\right)=S_{f}\left(X_{k}, X_{m}\right) \cdot S_{g}\left(X_{k}, X_{m}\right) \cdot S_{z}\left(X_{k}, X_{m}\right), \\
& S_{f}\left(X_{k}, X_{m}\right)=e^{-\left\|\frac{f_{k}-f_{m}}{h_{f}}\right\|^{2}}, S_{g}\left(X_{k}, X_{m}\right)=e^{-\left\|\frac{g_{k}-g_{m}}{h_{g}}\right\|^{2}}, S_{z}\left(X_{k}, X_{m}\right)=e^{-\left\|\frac{z_{k}-z_{m}}{h_{z}}\right\|^{2}}
\end{aligned}
$$

The parameters $h_{f}, h_{g}$ and $h_{z}$ are normalization factors which are user defined. Usually, these parameters express the scatter of vectors $f, g, z$, respectively.

From equation (2) we have

$$
S\left(X_{k}, X_{m}\right)=e^{-\left(\left\|\frac{f_{k}-f_{m}}{h_{f}}\right\|^{2}+\left\|\frac{g_{k}-g_{m}}{h_{g}}\right\|^{2}+\left\|\frac{z_{k}-z_{m}}{h_{z}}\right\|^{2}\right)}=e^{-\left\|X_{k}^{\prime}-X_{m}^{\prime}\right\|}
$$

where $X_{k}^{\prime}=\left[\frac{f 1_{k}}{h_{f}}, \frac{f 2_{k}}{h_{f}}, \frac{f 3_{k}}{h_{f}}, \frac{g 1_{k}}{h_{g}}, \frac{g 2_{k}}{h_{g}}, \frac{g 3_{k}}{h_{g}}, \frac{z 1_{k}}{h_{z}}, \frac{z 2_{k}}{h_{z}}\right]$ the properly normalized vector of $X_{k}$.

The proposed technique can be applied to color images without any sub-sampling. However, in the case of large-size images and in order to achieve reduction of the computational time and memory size requirements, it is preferable to have a subsampling version of the original image. We choose to use a fractal scanning process, based on the well-known Hilbert's space filling curve [10-11], where scans one area of the image completely before moving to the next. So, the neighborhood relationship between pixels is retained in neighboring samples.

\section{The SGONG Neural Network}

The proposed SGONG network consists of two separated layers of fully connected neurons, the input layer and the output mapping layer. In contrast with the Kohonen SOFM, the space of the mapping layer has always the same dimensionality with the input space, and also, the created neurons take their position in order the structure of neurons to approximate the structure of the input training vectors. In other words, the topology of the created neurons always approximates the topology of the training vectors. All vectors of the training data set $X^{\prime}$ are circularly used for the training of the SGONG network. In contrast with the GNG network, where a new neuron is 
always inserted at the end of each epoch, in the proposed algorithm three new criteria are applied that control the growing of the output lattice of neurons. In summary, we introduce the following three criteria:

- remove the inactive neurons,

- add new neurons,

- and finally, remove the non important neurons.

In accordance with the Kohonen SOFM, the proposed neural network uses the same equations for the adaptation of neuron's position and utilizes a cooling scheme for the learning rates used for weights readjustments. In contrast with the GNG classifier, in the proposed technique, the learning rates are locally defined in such a way that the adaptation ability of each neuron to each training vector is proportionally decreased with the number of vectors classified in the corresponding class. This removes the problem of increased neuron's plasticity or the danger of convergence in local minima, when the constant learning rates are not well adjusted. The proposed strategy makes the convergence faster, compared with the GNG classifier, as the weights of all neurons are stabilized and the network is forced to converge, when a predefined number of vectors have been classified to each neuron.

The adjacency relations between neurons are described by lateral connections between them. The Competitive Hebbian Rule (CHR) [12] is used to dynamically create or remove the lateral connections during the training procedure. This approach improves the data clustering capabilities of the SGONG network, in comparison with the Kohonen SOFM, where the lateral connections are fixed and predefined. Taking into account that the new neurons are inserted in order to support these with the highest error, and that the neuron's lateral connections perfectly describe the topology of the input vectors, we conclude that in contrast with Kohonen SOFM the proposed technique, during the training procedure, always gives a good description of the data structure. In addition, as the new neurons are inserted near these with the maximum accumulated error at the end of a single epoch, the proposed technique is robust to noisy data. The length of each epoch determines the robustness to noisy data. On the other hand, the convergence is not based on optimizing any model of the process or its data, as the proposed neural network shares almost the same weight-update scheme with the Kohonen SOFM neural network.

\subsection{The Training Steps of the SGONG Network}

The training procedure for the SGONG neural classifier starts by considering first two output neurons $(c=2)$. The local counters $N_{i}, i=1,2$ of created neurons are set to zero. The initial positions of the created output neurons, that is, the initial values for the weight vectors $W_{i}, i=1,2$ are initialized by randomly selecting two different vectors from the input space. All the vectors of the training data set $X^{\prime}$ are circularly used for the training of the SGONG network.

The training steps of the SGONG are as follows:

Step 1. At the beginning of each epoch the accumulated errors $A E_{i}^{(1)}, A E_{i}^{(2)}$, $\forall i \in[1, c]$ are set to zero. The variable $A E_{i}^{(1)}$ expresses, at the end of each epoch, the 
quantity of the total quantization error that corresponds to Neuron $_{i}$, while the variable $A E_{i}^{(2)}$, represents the increment of the total quantization error that we would have if the Neuron $_{i}$ was removed.

Step 2. For a given input vector $X_{k}$, the first and the second winner neurons Neuron $_{w 1}$, Neuron ${ }_{w 2}$ are obtained:

$$
\text { for } \text { Neuron }_{w 1}: S\left(X_{k}, W_{w 1}\right) \geq S\left(X_{k}, W_{i}\right) \forall i \in[1, c]
$$

$$
\text { for } \text { Neuron }_{w 2}: S\left(X_{k}, W_{w 2}\right) \geq S\left(X_{k}, W_{i}\right), \forall i \in[1, c] \text { and } i \neq w 1
$$

Step 3. The local variables $A E_{w 1}^{(1)}$ and $A E_{w 1}^{(2)}$ change their values according to the relations:

$$
\begin{gathered}
A E_{w 1}^{(1)}=A E_{w 1}^{(1)}+\left\|X_{k}^{\prime}-W_{w 1}^{\prime}\right\| \\
A E_{w 1}^{(2)}=A E_{w 1}^{(2)}+\left\|X_{k}^{\prime}-W_{w 2}^{\prime}\right\| \\
N_{w 1}=N_{w 1}+1
\end{gathered}
$$

Step 4. If $N_{w l} \leq N_{i d l e}$ then the local learning rates $\varepsilon 1_{w l}$ and $\varepsilon 2_{w l}$ change their values according to equations (8), (9) and (10). Otherwise, the local learning rates have the constant values $\varepsilon l_{w l}=\varepsilon 1_{\min }$ and $\varepsilon 2_{w 1}=0$.

$$
\begin{gathered}
\varepsilon 2_{w l}=\varepsilon 1_{w l} / r_{w l} \\
\varepsilon 1_{w l}=\varepsilon l_{\max }+\varepsilon l_{\min }-\varepsilon l_{\min } \cdot\left(\frac{\varepsilon l_{\max }}{\varepsilon l_{\min }}\right) \frac{N_{w l}}{N_{i d l e}} \\
r_{w l}=r_{\max }+1-r_{\max } \cdot\left(\frac{1}{r_{\max }}\right)^{\frac{N_{w l}}{N_{i d l e}}}
\end{gathered}
$$

The learning rate $\varepsilon l_{i}$ is applied to the weights of Neuron $_{i}$ if this is the winner neuron $(w 1=i)$, while $\varepsilon 2_{i}$ is applied to the weights of Neuron if $_{i}$ this belongs to the neighborhood domain of the winner neuron $(i \in \operatorname{nei}(w l))$. The learning rate $\varepsilon 2_{i}$ is used in order to have soft competitive effects between the output neurons. That is, for each output neuron, it is necessary that the influence from its neighboring neurons to be gradually reduced from a maximum to a minimum value. The values of the learning rates $\varepsilon l_{i}$ and $\varepsilon 2_{i}$ are not constant but they are reduced according to the local counter $N_{i}$. Doing this, the potential ability of moving of neuron $i$ toward an input 
vector (plasticity) is reduced with time. Both learning rates change their values from maximum to minimum in a period, which is defined by the $N_{\text {idle }}$ parameter. The variable $r_{w i}$ initially takes its minimum value $r_{\min }=1$ and in a period, defined by the $N_{\text {idle }}$ parameter, reaches its maximum value $r_{\max }$.

Step 5. In accordance with the Kohonen SOFM, the weight vector of the winner neuron Neuron $_{w 1}$ and the weight vectors of its neighboring neurons Neuron $_{m}$, $m \in n e i(w 1)$, are adapted according to the following relations:

$$
\begin{gathered}
W_{w 1}^{\prime}=W_{w 1}^{\prime}+\varepsilon 1_{w 1} \cdot\left(X_{k}^{\prime}-W_{w 1}^{\prime}\right) \\
W_{m}^{\prime}=W_{m}^{\prime}+\varepsilon 2_{m} \cdot\left(X_{k}^{\prime}-W_{m}^{\prime}\right), \forall m \in \operatorname{nei}(w l)
\end{gathered}
$$

Step 6. With regard to generation of lateral connections, SGONG employs the following strategy. The CHR is applied in order to create or remove connections between neurons. As soon as the neurons Neuron $_{w 1}$ and Neuron $_{w 2}$ are detected, the connection between them is created or is refreshed. That is

$$
s_{w 1, w 2}=0
$$

With the purpose of removing of superfluous lateral connections, the age of all connections emanating from Neuron $_{w 1}$, except the connection with Neuron $_{w 2}$, is increased by one:

$$
s_{w l, m}=s_{w 1, m}+1, \forall m \in n e i(w 1), \text { with } m \neq w 2
$$

Step 7. At the end of each epoch it is examined if all neurons are in idle state, or equivalently, if all the local counters $N_{i}, \forall i \in[1, c]$ are greater than the predefined value $N_{\text {idle }}$ and the neurons are considered well trained. In this case, the training procedure stops, and the convergence of SGONG network is assumed. The number of input vectors needed for a neuron to reach the "idle state" influences the convergence speed of the proposed technique. If the training procedure continues, the lateral connections between neurons with age greater than the maximum value $\alpha$ are removed. Due to dynamic generation or removal of lateral connections, the neighborhood domain of each neuron changes with time in order to include neurons that are topologically adjacent.

Step 8. At the end of each epoch, three criteria that modify the number of the output neurons $c$ and make the proposed neural network to become self-growing are applied. These criteria are applied in the following order:

- A class (neuron) is removed if for a predefined consecutive number of epochs, none of training samples have classified in this class.

- A new class (neuron) is added near the class with the maximum contribution in total quantization error (with the maximum $A E^{(1)}$ ), if the average distance of its vectors from neighboring classes is greater than a predefined value. This value is expressed as a percentage $\left(t_{1}\right)$ of the average distance between all classes. 
- The class (neuron) with the minimum average distance of its vectors from neighboring classes is removed if this quantity is less than a predefined value. This value is expressed as a percentage $\left(t_{2}\right)$ of the average distance between all classes.

In order to make faster the network convergence it can be defied not to apply the above criteria when the total number of epochs is above a predefined value. This has as a result the rapid passing of all neurons to the "idle state" and therefore the finalizing of the training procedure. After the training procedure the de-normalized vectors $W_{i}, i=1,2, \ldots c$ expresses the centers of final classes.

\section{On the Estimation of Most Important Classes}

As it is already mentioned, the EMIC technique requires only a predefined number of classes and a set of vectors representative of data space. It considers the position of given classes in feature space and the number of vectors classified to each class in order to choose automatically the most important classes. Initially the Comparative Hebbian Rule CHR [17] is applied extracting the lateral connections between classes. Then, we consider in the middle of each lateral connection a new class and we apply again the CHR in order to update the lateral connections between all classes. A kind of histogram is defined counting the population of vectors in connected nodes classes. The EMIC can be considered as finding the peaks in created histogram. A class is denoted as a peak if its height, i.e. the number of classified vectors, is greater of the height of neighboring classes. .

The Image in Fig. 1 is described in RGB color space and only for presentation reasons the color vectors are projected onto the plane defined by the first and the second Principal Component of all color vectors, Fig 1(b). Doing this, each color is represented by a two-dimensional vector. In Fig. 1(c) the main concentrations of 2Dvectors are described by white color. The SGONG classifier with proper settings converges to 16 classes whose centers and their neighboring relations are depicted on Fig. 1(d) with red circles and lines, respectively. We consider in the middle of each connection a new class and we continue the training procedure of SGONG, for only one epoch, considering that all classes are in idle state. This is happening in order to find again the new lateral connections using the CHR procedure that coexists in SGONG. A class is denoted as a peak if the number of vectors which have been classified to it is greater from the number of vectors classified to neighboring classes. As depicted in Fig. 1(e) 13 peaks have estimated which corresponds to the most important classes.

\section{Experiments}

The proposed method and the CQ methods that are based on the Kohonen SOFM, GNG, and FCM classifiers were implemented in software, called "ImageQuant", and can be downloaded and tested from the site http://ipml.ee.duth.gr/ papamark/ . In this, paper, due to the space limitation, we give only the following two experiments. Both experiments demonstrate the ability of the proposed SGONG neural classifier and the 


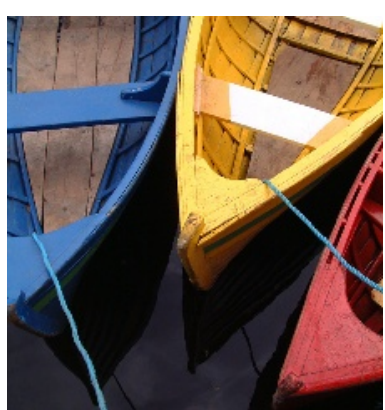

(a)

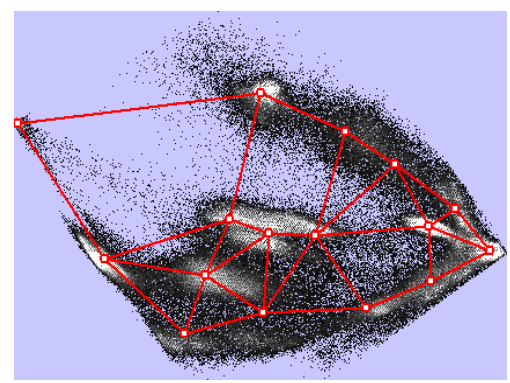

(d)

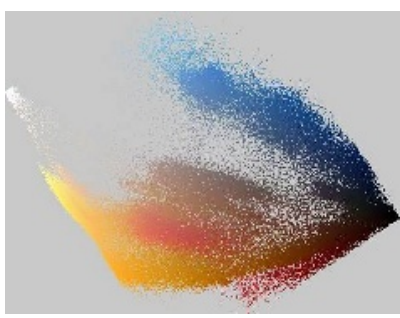

(b)

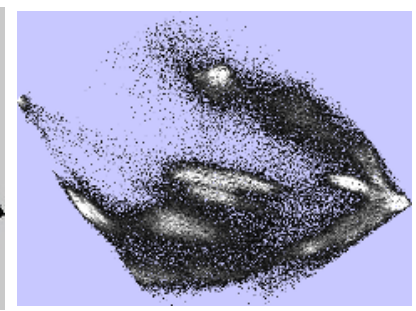

(c)

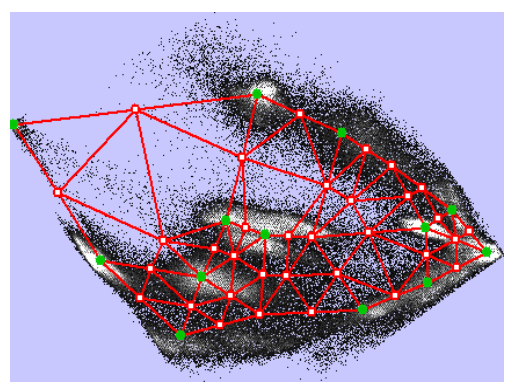

(e)

Fig. 1. Estimation of dominant colors

EMIC technique to define, in an adaptive way, the number of final classes, i.e. the number of final colors, according to the structure of the input data.

\section{Experiment 1}

In the first experiment the original image of Fig. 1 has 113081 unique colors. The proposed SGONG neural classifier converges to 16 unique colors applying the following main settings:

- The maximum number of output neurons is adjusted to 45 ,

- A new class (neuron) is added near this one with the maximum contribution in quantization error, if the average distance of its vectors from neighboring classes is greater than $30 \%$ of the average distance between all classes $\left(t_{1}=0.3\right)$.

- The class (neuron) with the minimum average distance of its vectors from neighboring classes is removed if this quantity is less than $20 \%$ of average distance between all classes $\left(t_{2}=0.2\right)$.

- The initial values for $\varepsilon 1_{\text {max }}, \varepsilon 1_{\text {min }}, r_{\text {max }}$ are set to $0.2,0.0005$ and 400 respectively.

- The original image is sub-sampled taking samples from the peaks of Hilbert's fractal. The size of fractal is adjusted to take almost 3000 samples. 
- A neuron is getting to "idle state" if 9000 vectors have classified to it $\left(N_{\text {idle }}=9000\right)$.

The number of most important classes obtained applying the EMIC technique. As depicted on Fig. 1(e) only 13 classes have estimated.

\section{Experiment 2}

In the second experiment the test image of Fig 2 has 33806 unique colors. In order to automatically find the number of the image dominant colors, the SGONG classifier uses the same settings as in Experiment 1. The resultant image is depicted on Fig 2 (b) and has only nine colors. The colors can be more reduced by applying the EMIC technique. Doing this, an image of only seven colors is constructed, which depicted on Fig 2(c). Furthermore, for comparison reasons, the CQ techniques based on Kohonen SOFM, the GNG, and the Fuzzy C-Means are applied. In order to have comparative results, the above techniques use exactly the same samples in the same order. Other applied techniques are the Color Quantization method of Dekker [13], and finally, the method of $\mathrm{Wu}$ [4]. In all above cases the RGB color model was used.

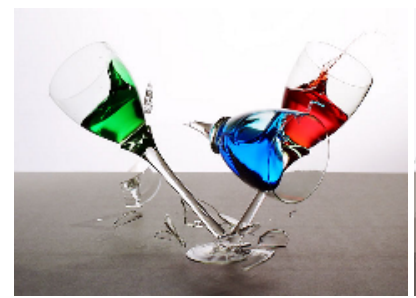

(a)

(d)

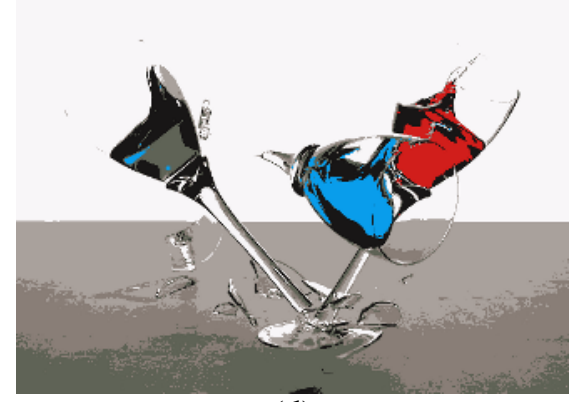

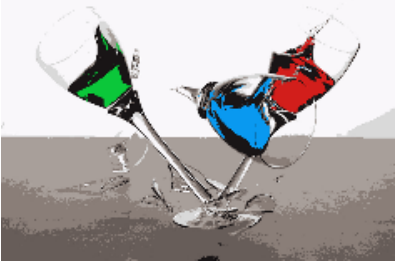

(b)

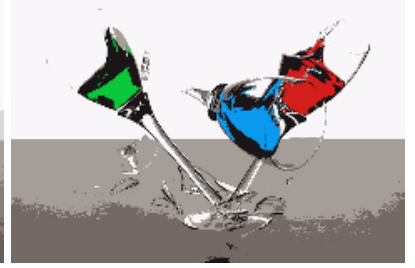

(c)

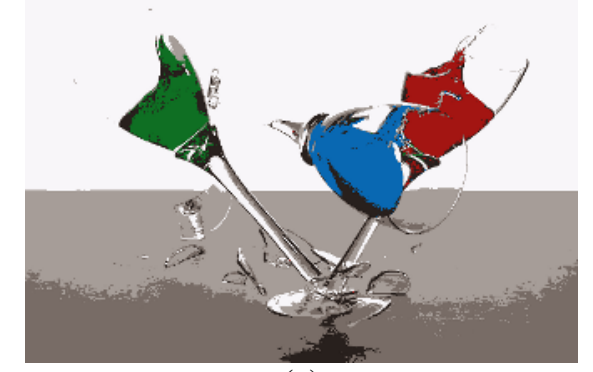

(e)

Fig. 2. (a) Initial image of 33806 unique colors. (b) Color quantization using the SGONG technique, the number of classes has automatically estimated to nine with appropriate settings. (c) The EMIC method is applied resulting in an image of only 7 classes. (d) Color quantization using the SGONG technique, the number of classes are predetermined and equal to 7 , the criteria that influence the growing of the output lattice of neurons are neglected. (e) The same settings with Fig (d) except of using with R,G,B color components the additional features "a" and " $b$ " of $\mathrm{L}^{*} \mathrm{a} * \mathrm{~b}$ * color space. 
Table 1. Comparative results

\begin{tabular}{|c|c|c|c|c|c|c|}
\hline & $\begin{array}{c}\text { SGONG \& } \\
\text { Estimation Fig.2(c) }\end{array}$ & $\begin{array}{c}\text { SGONG } \\
\text { Fig.2(d) }\end{array}$ & FCM & GNG & Dekker & Wu \\
\hline MSE & 694,28 & 719,38 & 1107,11 & 821,39 & 1972,72 & 1049,32 \\
\hline ADC & 42,51 & 44,29 & 52,52 & 46,6 & 77,21 & 54,21 \\
\hline SNR & 51,23 & 50,87 & 46,56 & 49,54 & 40,78 & 47,1 \\
\hline PSNR & 56,38 & 56,03 & 51,72 & 54,7 & 45,94 & 52,25 \\
\hline
\end{tabular}

\section{Conclusions}

This paper proposes a new CQ technique which is based on a new neural network classifier (SGONG). The SGONG network classifier is suitable for CQ applications. Each pixel is considered as a multidimensional vector which contains the color components and additional spatial characteristics derived from the neighborhood domain of each pixel. An efficient way to combine color and feature vectors is used. The main advantage of the SGONG network is that it controls the number of created neurons and their topology in an automatic way. . The convergence speed of SGONG classifier is comparable to the convergence speed of the GNG classifier, while the stability of SGONG classifier is comparable to the stability of Kohonen SOFM classifier. The number of resultant classes can efficiently be reduced more, applying the EMIC technique. The combination the SGONG and EMIC techniques in colored images enable the efficient description of images with very few numbers of colors. In order to speed up the entire algorithm, a fractal sub-sampling procedure based on the Hilbert's space filling curve is applied to initial image, taking samples only from the fractal peaks and their neighboring pixels. The proposed CQ technique has been extensively tested and compared to other similar techniques.

\section{References}

[1] Heckbert, P. (1982). Color image quantization for frame buffer display. Computer \& Graphics, 16:297-307.

[2] Wan, S.J., Prusinkiewicz, P. and Wong, S.K.M. (1990). Variance based color image quantization for frame buffer display. Color Research and Application, 15: 52-58.

[3] Ashdown, I. (1994). Octree CQ, from the book: Radiosity-A Programmer's Perspective, Wiley, New York.

[4] Wu, X. (1992). CQ by dynamic programming and principal analysis. ACM Transactions on Graphics, 11:384-372.

[5] Papamarkos, N., Atsalakis, A. and Strouthopoulos, C. (2002). Adaptive Color Reduction. IEEE Trans. On Systems, Man, and Cybernetics, Part B, 32:44-56.

[6] Baraldi, A. and Blonda, P. (1999). A Survey of Fuzzy Clustering Algorithms for Pattern Recognition-Part I\&II., IEEE Trans. On Systems, Man, and Cyb., Part B, 29:778-801. 
[7] Carpenter, G., Grossberg, S. and Rosen, D.B. (1991). Fuzzy ART: Fast stable learning and categorization of analog patterns by an adaptive resonance system. Neural Networks, 4:759-771.

[8] Bezdek, J.C. (1981). Pattern recognition with fuzzy objective function algorithms. Plenum Press, New York.

[9] Buhmann, J.M., Fellner, D.W., Held, M., Kettere, J. and Puzicha, J. (1998). Dithered CQ. Proceedings of the EUROGR. '98, Lisboa, Computer Graphics Forum 17(3): 219-231.

[10] Papamarkos, N. and Atsalakis, A. (2000). Gray-level reduction using local spatial features. Computer Vision and Image Understanding, 78:336-350.

[11] Papamarkos, N. (1999). Color reduction using local features and a SOFM neural network. Int. Journal of Imaging Systems and Technology, 10:404-409.

[12] Kohonen, T. (1990). The self-organizing map. Proceedings of IEEE, 78: 1464-1480.

[13] Dekker, A.H. (1994). Kohonen neural networks for optimal CQ. Computation in Neural Systems, 5:351-367.

[14] Huang, H.Y., Chen, Y.S. and Hsu, W.H. (2002). Color image segmentation using a selforganized map algorithm. Journal of Electronic Imaging, 11:136-148.

[15] Rahman, A. and Rahman, C.M. (2003). A new approach for compressing color images using neural network. Processing of CIMCA, pp. 12-14, Vienna, Austria.

[16] Fritzke, B. (1995). A growing neural gas network learns topologies. in Advances in Neural Information Processing Systems. 7, Tesauro, G., Touretzky, D.S. and Leen, T.K., Eds. Cambridge, MA: MIT Press, pp. 625-632.

[17] T. M. Martinetz and K. J. Schulten, "Topology representing networks," Neural Networks, vol. 7, no. 3, pp. 507-522, 1994.

[18] D. Comaniciu and P. Meer, "Mean Shift: A robust approach toward feature space analysis", IEEE Transactions on Pattern Analysis and Machine Intelligence, vol. 24, no. 5, 2002 . 